\title{
Influence de la variété et de la cuisson du pois (Pisum sativum) sur la nature des composés azotés des fèces de rats
}

\author{
Etiennette COMBE, Annick NAUDIN-LEBECQUE et R. PION \\ avec la collaboration technique de Marie-Claude Valluy, Françoise Barre, \\ M. Sallas et A. Selle \\ INRA, Laboratoire d'Etude du Métabolisme azoté, \\ Centre de Recherches de Clermont-Ferrand-Theix \\ Saint-Genès-Champanelle, F 63122 Ceyrat
}

\begin{abstract}
Résumé
Deux variétés de graines de Pois, Lincoln ou Frimas ont été utilisées comme seule source protéique dans la ration alimentaire équilibrée du rat en croissance. L'effet sur la digestion du traitement à l'autoclave de ces graines qui diffèrent par leurs teneurs en acides aminés et en glucides a été étudié. Bien que les CUDA de l'azote des graines crues des deux variétés soient voisines, les CUDA de la cystine, de la méthionine et du tryptophane (ainsi que la thréonine dans le cas des Lincoln) sont nettement plus bas que le CUDA de l'ensemble des AA( $\Sigma$ AA) et même que celui de l'Azote $(\mathrm{N})$. Les fèces des rats ayant reçu les régimes expérimentaux diffèrent par leur teneur en sucres aminés et par la composition en acides aminés des fractions éthanolosolubles ou acidosolubles. L'étude de la composition en acides aminés des 2 extraits solubles et du résidu des fèces montre que les phénomènes de digestion sont fortement influencées par la nature et l'état des glucides ingérés avec la source protéique. Les fractions solubles contiennent une faible proportion de l'azote fécal qui est très sensible à l'effet variété et à l'effet traitement des graines utilisées dans le régime. Il s'agit de composés en voie d'hydrolyse pouvant avoir une origine alimentaire ou endogène. La fraction insoluble des fèces dont la composition en acides aminés est peu modifiée par les facteurs étudiés correspond à un mélange de bactéries dont le développement semble favorisé dans le cas de l'utilisation de la variété Lincoln et dans le cas du traitement à l'autoclave.
\end{abstract}

Mots clés : Rat, digestion, acides aminés, pois, effet traitement

\section{Introduction}

Les graines de légumineuses sont riches en protéines, et leur teneur en lysine est satisfaisante, mais les teneurs en acides aminés soufrés, en tryptophane (Pois) ou en thréonine (Pois chiche) peuvent devenir insuffisantes pour satisfaire l'équilibre en acides aminés indispensables des régimes. Dans le cas de Pisum Sativum, les teneurs en protéines des graines varient de 17,25 à 32,2 p. 100 (MossÉ et al., 1987) selon les 
génotypes et les conditions de production. Pour Reichert \& MacKenzie (1982) les variations de la teneur en protéine des graines de la variété Trapper s'accompagnent de variations concomitantes de la teneur en amidon et pour une plus faible part des teneurs en fibre et en sucres. Par ailleurs, les graines de pois présentent un caractère lisse ou ridé associé à une structure et à une composition différentes de l'amidon (ColonNa \& Mercier, 1979). L'utilisation de ces graines dans l'alimentation est limitée par l'apparition de troubles digestifs attribués à certains de leurs composants glucidiques (FLEMING, 1981).

Nous avons voulu déterminer dans quelle mesure la digestion de la fraction azotée des graines était modifiée par leur composition relative en protéines et en glucides. Dans ce but, nous avons étudié l'effet du traitement préalable des graines sur la nature des composés azotés des fèces (séparés selon leur solubilité dans l'éthanol et dans l'acide trichloracétique) provenant de rats ayant ingéré les graines de pois de 2 variétés à haut rendement en grain sec et bonne teneur en protéines mais différentes par leur teneurs en glucides (Cerning-Beroard \& Filiatre-Verel, 1979) : les variétés de pois Frimas et Lincoln.

\section{Matériel et méthodes}

\section{A. Régimes alimentaires}

Leur composition est calculée de façon à satisfaire les besoins du rat en croissance en apportant 15 p. 100 de Matières Azotées Totales $(\mathrm{N} \times 6,25)$ et 1100 kilojoules par $\mathrm{kg}$ de matière sèche.

La seule source de protéines des régimes est constituée par des graines de pois de la variété Lincoln (Pois ridés) ou de la variété Frimas (Pois lisses) utilisées crues ou cuites (autoclave $120^{\circ} \mathrm{C}$ pendant 20 minutes). Les teneurs en matières azotées totales $(\mathrm{N} \times 6,25)$ et en acides aminés indispensables (sauf l'arginine) sont plus élevées dans les graines Lincoln que dans les graines Frimas (tabl. 1). Comparé aux besoins du rat en croissance, l'apport des graines dans le régime est carencé en méthionine (1,58 et $1,65 \mathrm{~g} / \mathrm{kg} \mathrm{MS}$ ). Ces deux variétés diffèrent aussi par leur composition en glucides et notamment en amylose, amylopectines, polyholosides et glucides solubles. Cependant, l'apport des graines assure 51 et 45 p. 100 de la ration énergétique pour les lots Frimas et Lincoln respectivement. De plus, ces graines apportent des teneurs en potassium non négligeables $(0,99$ et 1,32 p. $100 \mathrm{MS})$ mais des teneurs insuffisantes en Magnésium $(0,75$ et 0,93$)$ Phosphore $(2,5$ et 2,80$)$ et Calcium $(0,69$ et 0,93$)$.

Le régime a donc été complété comme indiqué dans le tableau 2, par une supplémentation en méthionine et tryptophane et par un mélange minéral adéquat. L'apport énergétique des graines a été complété par de l'amidon de blé et de l'huile. L'apport en oligo-éléments et vitamines a été assuré par les mélanges standards dont la composition est indiquée dans les notes du tableau 2.

Les constituants du régime, préalablement broyés, sont mélangés en milieu aqueux jusqu'à former une pâte homogène. La présence d'Agar-Agar permet d'obtenir une consistance évitant au maximum le gaspillage quand le régime est offert ad libitum aux rats chaque matin. 


\section{TABleAU 1}

Composition en acides aminés ( $\mathrm{g} / 16 \mathrm{~g} N$ ) des graines de pois ridés (Lincoln) et lisses (Frimas). Amino acid composition of wrinkled (Lincoln) and smooth (Frimas) peas.

\begin{tabular}{|c|c|c|}
\hline & Frimas & Lincoln \\
\hline $\begin{array}{l}\% \text { MS } / \% D M \\
(\mathrm{~N} \cdot 6.25) \% \mathrm{MS} /(\mathrm{N} \times 6.25) \% D M\end{array}$ & $\begin{array}{l}90,73 \\
23,88\end{array}$ & $\begin{array}{l}90,15 \\
27,31\end{array}$ \\
\hline 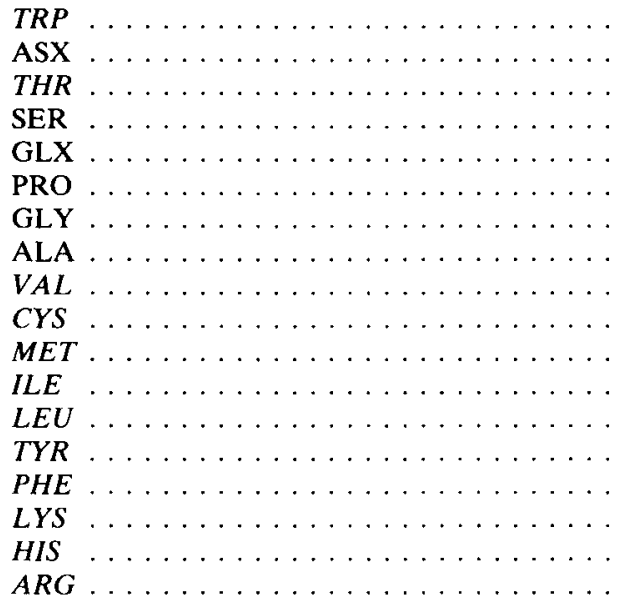 & $\begin{array}{r}0,84 \\
12,30 \\
3,80 \\
4,95 \\
17,90 \\
3,90 \\
4,35 \\
4,25 \\
4,70 \\
1,75 \\
1,05 \\
4,10 \\
6,90 \\
3,35 \\
4,05 \\
7,40 \\
2,40 \\
9,60\end{array}$ & $\begin{array}{r}0,86 \\
12,10 \\
4,20 \\
5,00 \\
17,50 \\
3,75 \\
4,65 \\
4,60 \\
5,00 \\
1,60 \\
1,10 \\
4,50 \\
7,05 \\
3,70 \\
4,75 \\
8,00 \\
2,35 \\
8,15\end{array}$ \\
\hline 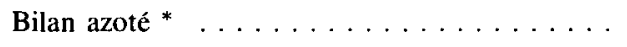 & 86,6 & 85,91 \\
\hline AAI $\ldots \ldots \ldots \ldots$ & 49,94 & 51,26 \\
\hline$\Sigma$ AA & 97,59 & 98,86 \\
\hline
\end{tabular}

\section{B. Animaux}

20 rats mâles de race Sprague Dawley âgés de 21 jours ont été placés en cage grillagée individuelle en raterie éclairée de 7 à 19 heures, maintenue à 60 p. 100 d'hygrométrie et à $22^{\circ} \mathrm{C}$. Ils ont reçu un régime standard pendant une semaine au bout de laquelle ils ont été répartis en 4 lots homogènes en fonction de leur poids vif et de leur vitesse de croissance. Ils ont alors reçu l'un des 4 régimes expérimentaux pendant 15 jours consécutifs, le bilan des quantités ingérées et excrétées a été effectué au cours des 5 derniers jours. La matière sèche ingérée était déterminée chaque jour pour chaque animal. Les fèces collectées quantitativement étaient stockées à $-15^{\circ} \mathrm{C}$ de façon à disposer en fin de période de la totalité des fèces émises par chaque animal. A la fin de la période expérimentale, le matin avant la distribution du repas, les rats ont été anesthésiés à l'éther et sacrifiés après que l'on ait prélevé et pesé le cœcum plein. 


\section{TABLEAU 2}

Composition des régimes $(\mathrm{g} / \mathrm{kg})$.

Practical diet composition.

\begin{tabular}{|c|c|c|}
\hline & Frimas & Lincoln \\
\hline Pois / Peas & 628,20 & 549,30 \\
\hline Amidon blé / Wheat starch & 164,80 & 234,60 \\
\hline Agar Agar & 30 & 30 \\
\hline Prémélanges / Premix ${ }^{(1+2)}$ & 36,2 & 36,2 \\
\hline Prémélange macroéléments & 36,2 & 36,2 \\
\hline Huile arachide / Peanut oil & 80,4 & 89,4 \\
\hline Huile maïs vitaminée ${ }^{(3)} /$ Maize oil + vit & 20 & 20 \\
\hline Biotine Choline $^{(4)} \ldots \ldots \ldots \ldots \ldots \ldots$ & $10 \mathrm{ml}$ & $10 \mathrm{ml}$ \\
\hline
\end{tabular}

Suppléments apportés par le prémélange macroéléments $(36,2 \mathrm{~g})$

\begin{tabular}{|c|c|c|}
\hline MET $\ldots \ldots \ldots \ldots \ldots \ldots \ldots \ldots \ldots$ & 1,69 & 1,85 \\
\hline TRP $\ldots \ldots \ldots \ldots \ldots \ldots$ & 0,24 & 0,21 \\
\hline 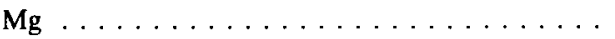 & 0,25 & 0,07 \\
\hline$\ldots \ldots$ & 2,0 & 2,0 \\
\hline$\ldots \ldots$ & 4,49 & 4,20 \\
\hline $\mathrm{Ca} \ldots \ldots \ldots \ldots \ldots \ldots \ldots$ & 9,31 & 9,07 \\
\hline
\end{tabular}

(1) Mélange oligo-éléments $18,1 \mathrm{~g}$ (composition $/ \mathrm{kg}$ MS : citrate $\mathrm{NH}_{4}$, Fe III $343 \mathrm{mg} ; \mathrm{Mn} \mathrm{CO}, 83,6 \mathrm{mg}$; $\mathrm{CuSO}_{4}, 5 \mathrm{H}_{2} \mathrm{O} 39,4 \mathrm{mg}$; $\mathrm{Zn} \mathrm{O} \mathrm{IV,} 7 \mathrm{H}_{2} \mathrm{O} 132 \mathrm{mg}$; $\mathrm{NaF} 11 \mathrm{mg} ; \mathrm{KI} \mathrm{0,26} \mathrm{mg;} \mathrm{CoCO}_{3} 0,20 \mathrm{mg} ; \mathrm{SeO}_{2} 0,14 \mathrm{mg}$; $\mathrm{Al}_{2}\left(\mathrm{SO}_{4}\right)_{3} \mathrm{Kr} \mathrm{SO} \mathrm{SO}_{4}, 24 \mathrm{H}_{2} \mathrm{O} 1,75 \mathrm{mg}$; amidon qsp).

(2) Mélange vitamines $18,1 \mathrm{~g}$ (composition $/ \mathrm{kg}$ MS : $\mathrm{B}_{12}: 3 \mathrm{mg}$; Acide folique $0,1 \mathrm{mg} ; \mathrm{E}: 3 \mathrm{~g} ; \mathrm{K}_{1}: 0,5 \mathrm{~g} ; \mathrm{B}_{1}$ : $0,8 \mathrm{~g}$; acide nicotinique : $3 \mathrm{~g} ; \mathrm{B}_{6}: 0,8 \mathrm{~g} ; \mathrm{B}_{2}: 1,5 \mathrm{~g}$; Pantothénate de calcium : $3 \mathrm{~g}$; cyclohexane hexol $50 \mathrm{~g}$; Acide para-amino-benzoïque : $50 \mathrm{~g}$; amidon $\mathrm{qsp}$ ).

(3) Rétinol (A) et ergocalciférol (D2) sont ajoutés dans l'huile de maïs (20000 et $10000 \mathrm{IU} / 100 \mathrm{ml}$ ).

(4) $4,5 \mathrm{ml}$ Solution aqueuse de biotine $(4 \mathrm{mg} / 100 \mathrm{ml}) 5 \mathrm{ml}$ solution aqueuse de choline $(20000 \mathrm{IU} / 100 \mathrm{ml})$.

\section{Traitement des fèces}

Les fèces congelées sont lyophilisées. Les fèces provenant des rats d'un même lot sont ensuite mélangées et passées au broyeur à billes. Les traitements ultérieurs sont effectués sur des quantités aliquotes d'un poids suffisant pour permettre une détermination aisée des composés azotés solubles.

- Extractions des composés solubles des fèces et filtration sur gel : les composés solubles dans l'éthanol 80 p. 100 sont obtenus par 3 extractions successives à $-10^{\circ} \mathrm{C}$ dans un volume $(\mathrm{ml})$ d'alcool 80 p. 100 égal à 40 fois le poids $(\mathrm{g})$ de l'échantillon fécal sec. Les résidus sont séparés des extraits solubles par centrifugation à $-10^{\circ} \mathrm{C}$ à $5500 \mathrm{~g}$. L'ensemble des surnageants regroupés constitue l'extrait éthanolique.

Le résidu de cette extraction est ensuite traité de la même façon avec de l'acide trichloroacétique (ATC) $10 \mathrm{p} .100$ à $+4^{\circ} \mathrm{C}$ pour extraire les composés solubles dans l'ATC.

On obtient ainsi d'abord les composés solubles dans l'éthanol 80 p. 100 et ensuite les composés insolubles dans l'éthanol resolubilisés par l'ATC 10 p. 100. Ces 2 extraits 
sont ensuite traités de façon identique afin d'estimer la taille et la composition des molécules azotées qu'ils renferment.

Après concentration, les extraits sont directement filtrés sur Séphadex G25. Les composés élués par CINa 0,2 p. $100(25 \mathrm{ml} / \mathrm{h})$ sont recueillis sur collecteur par fractions de $5 \mathrm{ml}$. Les fractions contenant des peptides ou des acides aminés libres sont repérées par enregistrement du spectre d'absorption en ultra-violet à $280 \mathrm{~nm}$ en continu et par lecture de la Densité-Optique à $570 \mathrm{~nm}$, après réaction à la ninhydrine dans chaque tube. Les tubes correspondant à chaque pic sont regroupés. Ceux-ci sont identifiés par les numéros des tubes constituant le début et la fin de chaque pic.

- Résidus insolubles: après rinçage et séchage à l'éthanol 100 et à l'éther sulfurique les résidus sont séchés avant d'être broyés.

- Dosages : l'azote est déterminé par la méthode Kjeldahl, les acides aminés sont dosés après hydrolyse par $\mathrm{H} \mathrm{Cl} 6 \mathrm{~N}$ par chromatographie sur résine échangeuse de cations à l'aide d'un appareil automatique selon une technique dérivée de celle de Moore et al. (1958). Les acides aminés soufrés sont dosés après oxydation performique et le tryptophane selon la méthode décrite par SLump et Schreuder (1969). Les sucres aminés sont dosés après hydrolyse ménagée, chromatographie sur échangeur de cations comme décrit par Combe et al. (1980). L 'azote aminé des fractions solubles séparées sur G 25 est dosé par réaction colorée à la ninhydrine avant et après hydrolyse acide. Le rapport des 2 concentrations ( $\mathrm{N}$ aminé total/ $\mathrm{N}$ aminé libre) est utilisé pour évaluer le nombre moyen de résidus d'acides aminés présents dans chaque pic.

\section{Modes d'expression des résultats}

Les résultats des analyses d'azote sont rapportés aux fèces sèches et exprimés en matières azotées totales $(\mathrm{MAT}=\mathrm{N} \times 6,25)$. Les résultats des analyses des sucres aminés, du tryptophane et des autres acides aminés sont exprimés en g/16 g d'azote (ou $100 \mathrm{~g}$ de MAT). Le résultat du dosage de l'azote aminé par colorimétrie dans les fractions solubles est exprimé en $\mathrm{g} / 100 \mathrm{~g}$ fèces lyophilisées. Les pourcentages de l'azote des acides aminés et des sucres aminés dosés dans l'azote total des fèces, des fractions solubles et des résidus constituent les «bilans azotés» des analyses.

Sur les tableaux et figures les 18 Acides Aminés (AA) dosés sont désignés par leur abréviation conventionnelle. On utilise aussi AAI ou AAE pour désigner le total des Acides Aminés Indispensables et $\Sigma$ AA pour la somme des Acides Aminés dosés.

Les différences de composition en acides aminés de 2 protéines sont étudiées en comparant les teneurs de chaque acide aminé $(\mathrm{g} / 16 \mathrm{~g} \mathrm{~N})$ dans ces 2 protéines. Nous avons aussi utilisé le calcul de l'Ecart Relatif Moyen (ERM) qui est la moyenne des écarts relatifs entre les teneurs en chacun des acides aminés qui composent ces protéines :

$E R M=100 \frac{1}{n} \Sigma n \sqrt{(A A i k-A A j k)^{2}} / \frac{\text { AAik }+ \text { AAjk }}{2}$

où $\mathrm{n}$ est le nombre d'acides aminés pris en considération dans la protéine, AAik et AAjk sont les pourcentages respectifs de l'acide aminé $k$ dans les protéines i et $\mathrm{j}$ que l'on veut comparer. Les valeurs obtenues sont d'autant plus faibles que les compositions des protéines qui font l'objet du calcul sont proches (PIon et al 1977). Les comparai- 
sons sont effectuées entre les différentes fractions des fèces d'un même lot; pour chaque fraction nous avons décrit l'effet variété en comparant les Lincoln aux Frimas et pour chaque variété, l'effet du traitement hydrothermique.

Analyses statistiques : la comparaison des moyennes a été effectuée par le test $t$ de Student après analyse de variance.

\section{Résultats}

\section{A. Contenus digestifs}

Les contenus de cœecum des rats ayant ingéré les pois Lincoln ridés atteignent 136 p. 100 du poids des cœecum des rats ayant ingéré les pois Frimas lisses. Le traitement à l'autoclave n'entraîne pas d'augmentation importante du poids moyen des contenus de cœcum : 103 p. 100 pour le lot Frimas et 101 p. 100 pour le lot Lincoln.

L'utilisation des régimes à base de Pois Lincoln, comparée à l'utilisation des Pois Frimas, entraîne une accumulation plus importante de matériel non digéré au niveau du cœcum. Ce phénomène n'est pas atténué par l'utilisation des graines cuites.

\section{B. Utilisation digestive apparente}

Les quantités de matière sèche ingérée (MSI) moyennes par jour et par animal entre lots cuits et crus ne sont pas significativement différentes, au seuil 0,01 (tabl. 3).

L'analyse de variance des quantités moyennes de matière sèche (MSF) excrétées dans les fèces par animal fait apparaître un effet des 2 facteurs étudiés : variété et traitement. La matière sèche excrétée dans les fèces est plus élevée dans le cas du lot Lincoln que dans le cas du lot Frimas. Le traitement à l'autoclave a pour effet d'augmenter la différence entre les deux lots $(\mathbf{P}<0.01)$.

Les cœfficients d'utilisation digestive apparente (CUDA) de la matière sèche et de l'énergie sont un peu plus faibles $(-2,5$ p. 100) dans le cas de la variété Lincoln que dans le cas de la variété Frimas. Par contre le CUDA des matières azotées est égal à 85 p. 100 pour les 2 variétés utilisées à l'état cru.

Le traitement hydrothermique a pour effet de diminuer la valeur de ces cœfficients notamment pour le CUDA de la MA et de façon plus importante dans le cas des Lincoln ( -10 p. 100) que Frimas ( -2 p. 100). En outre, les CUDA des graines cuites des 2 variétés diffèrent de 8 p. 100 pour MS et de 7 p. 100 pour l'énergie et les M.A.

\section{Composés azotés des fèces}

\section{Fèces}

a) Matières azotées (MA): les teneurs en matières azotées des fèces (fig. 1) provenant des rats ayant ingéré les pois Lincoln sont moins élevées que celles provenant du lot ayant ingéré les pois Frimas crus. Dans le cas des lots ayant ingéré les 
graines cuites, les teneurs en matières azotées des fèces sont augmentées et la différence entre Lincoln et Frimas légèrement atténuée. Cependant, les quantités moyennes de matières azotées excrétées par jour et par animal sont peu différentes. Ceci correspond au résultat présenté dans le paragraphe précédent pour le CUDA des matières azotées.

\section{TABleau 3}

Digestibilité de la matière sèche, de l'énergie et des matières azotées Dry matter, Energy and Nitrogen balances.

\begin{tabular}{|c|c|c|c|c|}
\hline & \multicolumn{2}{|c|}{ Frimas } & \multicolumn{2}{|c|}{ Lincoln } \\
\hline & Cru / Raw & Cuit / Cooked & Cru / Raw & Cuit / Cooked \\
\hline$\cdots \cdots \cdots \cdots$ & $16,44 \pm 0,55^{\mathrm{a}}$ & $15,61 \pm 0,55^{\mathrm{a}}$ & $17,20 \pm 0,70^{\mathrm{b}}$ & $17,78 \pm 0,67^{\mathbf{b}}$ \\
\hline$\overline{\mathrm{MSF}}$ & $1,98 \pm 0,13^{a}$ & $2,10 \pm 0,14^{b}$ & $2,51 \pm 0,25^{c}$ & $3,64 \pm 0,16^{d}$ \\
\hline CUDA $(\%)$ & & & & \\
\hline $\begin{array}{l}\text { MS } \ldots \ldots \ldots \ldots \\
\text { Energie } \ldots \ldots \ldots \ldots \\
\text { MA } \ldots \ldots \ldots \ldots \ldots\end{array}$ & $\begin{array}{l}87,9 \\
88,8 \\
85\end{array}$ & $\begin{array}{l}86,5 \\
87,7 \\
82,6\end{array}$ & $\begin{array}{l}85,4 \\
86,7 \\
85,1\end{array}$ & $\begin{array}{l}79,5 \\
81,5 \\
76,6\end{array}$ \\
\hline
\end{tabular}

MSI et MSF = quantités moyennes de matières sèches (I : ingérées, F : excrétées dans les fèces) g/jour et par animal, écart-type de la moyenne, 5 rats par lot.

a, b, c, d les moyennes d'une même ligne affectée de la même lettre ne sont pas significativement différentes au seuil 0,01 .

Les Coefficients d'Utilisation Digestive Apparente (CUDA) sont calculés selon la formule (Ingéré - Excrété fèces) $100 /$ Ingéré.

$\mathrm{Ng} / 100 \mathrm{~g}$ tèces



cru

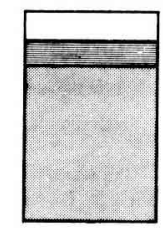

cuit



soluble éthanol

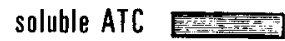
insoluble
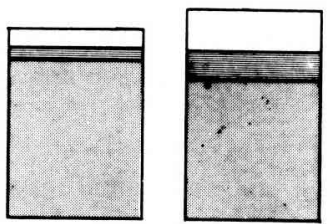

cru cuit

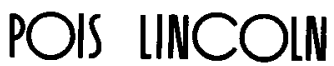

Fig. 1

Teneur en azote des fèces, répartition selon la solubilité dans l'éthanol et l'ATC. Soluble and insoluble nitrogen concentration in the faeces of rats fed the 4 diets. 
b) Sucres aminés : la teneur en glucosamine des matières azotées des fèces des rats ayant ingéré les graines crues est en moyenne de 3,5 g/100 g MAT (tabl. 4). Comparée à celles-ci, la teneur en galactosamine des matières azotées des fèces est deux fois et demi plus faible. Le traitement entraîne une augmentation de 43 et 31 p. 100 des teneurs en galactosamine et glucosamine respectivement dans le cas des lots Frimas. Dans le cas des lots Lincoln, le traitement entraîne une augmentation importante $(+55$ p. 100) de la seule galactosamine. En conséquence, les fèces provenant du lot ayant ingéré les Lincoln cuits présentent un rapport glucosamine/galactosamine nettement plus faible $(1,6)$ que ceux provenant des autres lots $(2,6$ à 2$)$.

c) Acides Aminés. Le total des teneurs ( $\mathrm{g} / 16 \mathrm{~g} \mathrm{~N}$ ) des 18 acides aminés dosés dans les fèces $(\Sigma \mathrm{AA})$ est plus faible pour la variété Lincoln que pour la variété Frimas. Il diminue sous l'effet du traitement. Ces différences sont plus marquées si on considère la somme des 12 acides aminés indispensables (AAI).

Le bilan azoté des acides aminés et sucres aminés montre que les MAT des fèces contiennent 29 et 35 p. 100 de composés azotés non dosés. La présence de ces composés semble augmentée par l'utilisation des pois Lincoln et par la cuisson des graines.

Compte tenu de la teneur en azote des fèces, on peut dire que l'utilisation des Pois Lincoln se traduit par une diminution de la concentration de tous les acides aminés fécaux. La cuisson entraîne des effets similaires (sauf dans le cas des acides dicarboxyliques).

\section{Comparaisons avec l'aliment}

a) Les teneurs en tryptophane, thréonine, alanine, cystine et méthionine sont toujours plus élevées dans les matières azotées des fèces (tabl. 4) que dans celles des graines (tabl. 1). De plus, dans le cas de l'ingestion des graines Frimas les teneurs en valine et isoleucine des MAT des fèces sont plus élevées que celles des graines. Dans le cas de l'ingestion des graines cuites Frimas, la glycine se trouve aussi en teneur plus élevée dans les MAT des fèces que dans celles des graines. Par contre les teneurs en arginine, histidine, lysine et leucine sont toujours nettement plus faibles dans les matières azotées des fèces que dans celles des graines.

b) Les écarts relatifs moyens (ERM) de la composition en acides aminés des matières azotées des fèces des 4 lots, entre eux et avec une estimation de la composition en acides aminés d'un mélange de bactéries des fèces du monogastrique (MASON et al. 1976), de l'endogène digestif (résidu des contenus de coecum de rats axéniques ayant ingéré un régime à 4 p. 100 de protéines d'œuf; CомвE et al. 1970) et de l'aliment (composition en acides aminés des graines des variétés de Pois utilisées) sont présentés dans le tableau 5. Les compositions en acides aminés des fèces des 4 groupes sont très voisines, notamment pour les 2 groupes ayant ingéré les graines cuites. Elle diffère un peu plus de la composition en acides aminés de l'aliment (ERM $=30$ à 35) que de celle des autres composés étudiés : endogène (ERM $=22$ à 29), bactéries (ERM $=20$ à $35)$. Les écarts de composition des fèces et de l'ingéré sont semblables $(E R M=31)$ dans le cas de l'ingestion des graines crues des 2 variétés. Par contre l'écart est légèrement augmenté dans le cas de l'utilisation des graines cuites de la variété Lincoln. Dans le cas des 2 variétés l'écart entre les fèces et les bactéries augmente sous l'effet du traitement. L'écart entre fèces et endogène est moins sensible aux facteurs étudiés. 


\section{TABLEAU 4}

Sucres aminés et acides aminés des fèces ( $\mathrm{g} / 16 \mathrm{~g} \mathrm{~N})$.

Faecal amino sugars and Amino acids.

\begin{tabular}{|c|c|c|c|c|}
\hline & \multicolumn{2}{|c|}{ Frimas } & \multicolumn{2}{|c|}{ Lincoln } \\
\hline & Cru / Crude & Cuit / Cooked & Cru / Crude & Cuit / Cooked \\
\hline$(\mathrm{N} \cdot 6,25) \% \mathrm{MS} \quad \ldots$. & 19,9 & 21,0 & 16,2 & 18,7 \\
\hline $\begin{array}{l}\text { Glucosamine } \ldots \ldots \ldots \\
\text { Galactosamine } \ldots \ldots\end{array}$ & $\begin{array}{l}3,4 \\
1,3\end{array}$ & $\begin{array}{l}4,6 \\
2,3\end{array}$ & $\begin{array}{l}3,6 \\
1,45\end{array}$ & $\begin{array}{l}3,6 \\
2,2\end{array}$ \\
\hline  & $\begin{array}{c}1,35 \\
8,8 \\
5,0 \\
3,95 \\
10,35 \\
3,75 \\
4,6 \\
6,8 \\
5,45 \\
2,50 \\
2,70 \\
4,35 \\
6,05 \\
3,40 \\
6,30 \\
1,6 \\
3,70 \\
47,5 \\
85,5\end{array}$ & $\begin{array}{r}1,15 \\
9,40 \\
4,75 \\
3,70 \\
10,20 \\
3,35 \\
4,45 \\
5,65 \\
4,20 \\
2,00 \\
1,45 \\
3,35 \\
4,95 \\
2,95 \\
5,15 \\
1,50 \\
3,30 \\
37,65 \\
74,39\end{array}$ & $\begin{array}{l}1,25 \\
8,45 \\
5,0 \\
3,85 \\
9,65 \\
3,30 \\
4,55 \\
6,35 \\
4,80 \\
2,20 \\
1,95 \\
3,80 \\
5,50 \\
3,20 \\
5,70 \\
1,45 \\
3,60 \\
41,75 \\
77,9\end{array}$ & $\begin{array}{r}1,1 \\
9,15 \\
4,50 \\
3,55 \\
9,85 \\
3,15 \\
4,50 \\
5,80 \\
3,85 \\
2,05 \\
1,50 \\
3,05 \\
4,60 \\
2,75 \\
5,05 \\
1,40 \\
2,95 \\
35,45 \\
71,45\end{array}$ \\
\hline $\begin{array}{l}\text { Bilan azoté } \\
\% \text { AAI } / \Sigma \text { AA }\end{array} \ldots \ldots$ & $\begin{array}{l}70,8 \\
55,5\end{array}$ & $\begin{array}{l}62,0 \\
50,59\end{array}$ & $\begin{array}{l}65,2 \\
53,59\end{array}$ & $\begin{array}{l}59,5 \\
49,62\end{array}$ \\
\hline
\end{tabular}

* 100 (N $\Sigma$ AA + amino sugars)/Kjeldahl.

\section{Digestibilité apparente des acides aminés (fig. 2)}

Le CUDA du total des AA est toujours plus élevé que celui de l'azote, mais il varie dans le même sens sous l'influence des facteurs étudiés. La différence entre les CUDA de l'azote et des AA passe de 2,5 à 4,$7 ; 6,1$ et 11,5 p. 100 respectivement pour les graines de Frimas crues et cuites et pour les Lincoln crues et cuites. Le CUDA des AAI est un peu plus faible que celui du total des A.A. Il n'est pas modifié par le traitement dans le cas des Frimas mais il diminue de 3,6 p. 100 dans le cas des Lincoln.

Les valeurs extrêmes du CUDA des AAI individuels concernent dans tous les cas pour les valeurs les plus basses la cystine, la méthionine et le tryptophane (ainsi que la thréonine dans le cas des Frimas) et l'Arginine et l'Histidine pour les valeurs les plus élevées (ainsi que la thréonine dans le cas des Lincoln). 


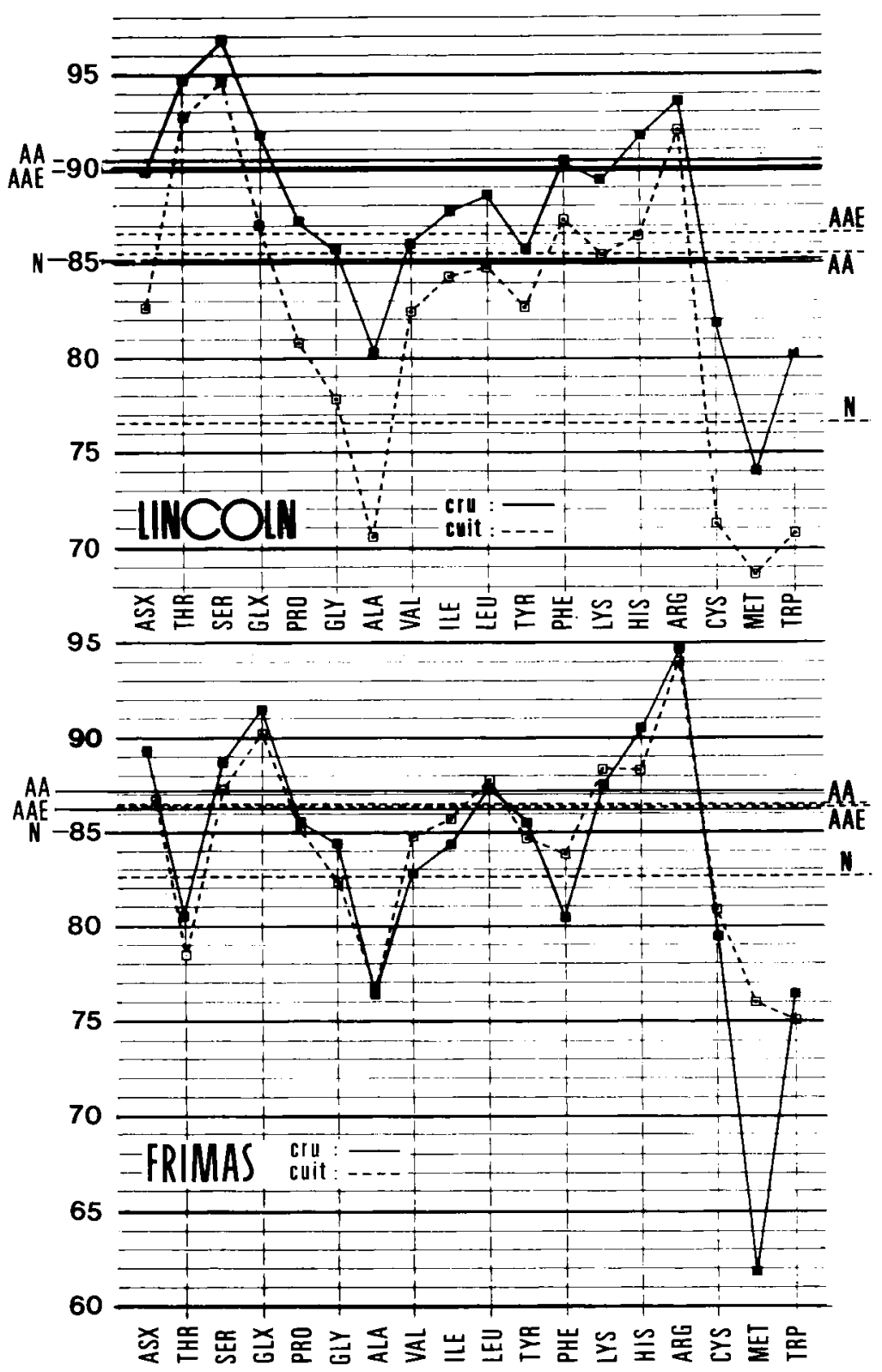

FIG. 2

Comparaison du CUDA des 18 Acides aminés dosés, selon la variété et le traitement des graines de Pois du régime.

Amino acids and Nitrogen apparent digestibility Coefficients (ADC) as affected by variety and cooking of peas.

Dans chaque cas les lignes horizontales indiquent le CUDA de l'azote total (N) d'une part et d'autre part la valeur moyenne calculée pour l'ensemble des Acides aminés ( $\Sigma$ AA) et pour les Acides aminés Indispensables. Le cas des Lincoln est représenté dans la partie supérieure et celui des Frimas dans la partie inférieure de la figure. Les lignes (carrés) sont pleines (noires) dans le cas des graines cuites et vides (blanches) dans le cas des graines crues.

In each case Nitrogen, $\Sigma A A, E A A$ coefficients are quoted by the horizontal lines; Lincoln are represented in the upper, and Frimas in the lower part of the figure; the raw/cooked factors are represented by empty/full lines or squares. 


\section{TABleau 5}

Ecart relatif moyen (ERM) de la composition en acides aminés des fèces entre les lots et avec des protéines de référence.

Amino Acids Average Relative Difference (ARD) between faeces from experimental pools and reference proteins $\left(n^{\circ} 1\right.$ : faeces from rats fed the raw Frimas diet; $n^{\circ} 2:$ faeces from rats fed cooked Frimas; $n^{\circ} 3$ : faeces from rats fed raw Lincoln; $n^{\circ} 4$ : faeces from rats fed cooked Lincoln; $n^{\circ} 5:$ bacterias; $n^{\circ} 6$ : endogenous material ; $n^{\circ} 7:$ ingested peas).

\begin{tabular}{|c|c|c|c|c|c|}
\hline & $\mathrm{N}^{o s}$ & 1 & 2 & 3 & 4 \\
\hline Fèces Frimas cru & 1 & 0 & & & \\
\hline Fèces Frimas cuit & 2 & 13 & 0 & & \\
\hline Fèces Lincoln cru & 3 & 9 & 9 & 0 & \\
\hline Fèces Lincoln cuit & 4 & 20 & 5 & 12 & 0 \\
\hline Mélange bactéries ...... & 5 & 20 & 31 & 26 & 35 \\
\hline Mélange endogène & 6 & 29 & 22 & 25 & 24 \\
\hline Graines Pois ingérées . . & 7 & 31 & 30 & 31 & 35 \\
\hline
\end{tabular}

On obtient des valeurs $<10$ lorsque l'on calcule l'ERM des acides aminés de fèces de lots de rats ayant ingéré des régimes de même composition.

ARD values fall below 10 when calculated out of samples from rats fed on diets of the same composition.

L'effet variété se manifeste pour la thréonine, la sérine, l'alanine, la valine, la méthionine et la phénylalanine par une augmentation de +8 à $17 \mathrm{p} .100$ des valeurs des CUDA dans le cas de l'utilisation de la variété Lincoln à la place de la variété Frimas.

La cuisson se manifeste, dans le cas de la variété Lincoln, par une diminution du CUDA de tous les acides aminés et notamment de la cystine ( -13 p. 100), du tryptophane $(-11,6$ p. 100), de la glycine $(-8,3$ p. 100) et de la méthionine $(-8$ p. 100). Dans le cas de la variété Frimas, la cuisson n'entraîne que de faibles variations des CUDA des AA sauf dans le cas de méthionine (+ 23 p. 100).

\section{Composés azotés insolubles des fèces}

La proportion des matières azotées insolubles est toujours très élevée dans les fèces : elle dépasse 80 p. 100 dans le cas des fèces des rats ayant ingéré les graines crues. Dans le cas des lots ayant ingéré les graines cuites, elle tombe à 75 et 67 p. 100 respectivement pour les variétés Frimas et Lincoln (fig. 1).

Les teneurs en acides aminés des matières azotées insolubles fécales sont élevées (tabl. 6). Elles sont toujours supérieures à celles des MAT fécales sauf pour le tryptophane dans tous les cas et pour la proline et la méthionine dans le cas du lot de rats ayant ingéré les Frimas crus. Le bilan de l'azote montre que les matières azotées insolubles contiennent 26 à 19 p. 100 de composés azotés non dosés, ce qui est inférieur aux proportions trouvées dans les fèces totaux. Les teneurs des AA des MAT insolubles varient selon le lot considéré. Quand on passe de l'utilisation de la variété Frimas à celle de la variété Lincoln, sous forme crue, on observe une diminution du total des teneurs des 18 AA et pour une moindre part des AAI. Cependant, la teneur 
TABLEAU 6

Acides aminés insolubles des fèces $(\mathrm{g} / 16 \mathrm{gN})$.

Faecal insoluble Amino acids.

\begin{tabular}{|c|c|c|c|c|}
\hline & \multicolumn{2}{|c|}{ Frimas } & \multicolumn{2}{|c|}{ Lincoln } \\
\hline & Cru / Crude & Cuit / Cooked & Cru / Crude & Cuit / Cooked \\
\hline$(\mathrm{N} \times 6,25) \% \mathrm{MS}$ insoluble & 19,69 & 15,29 & 15,25 & 12,68 \\
\hline 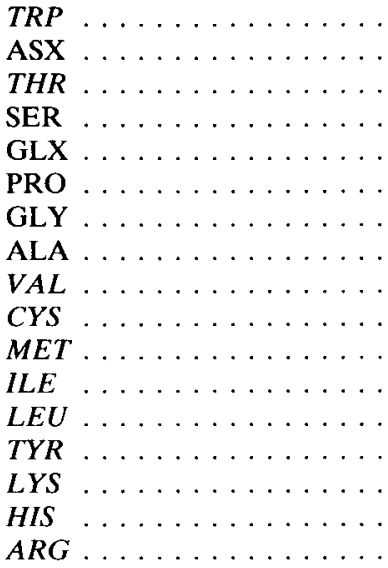 & $\begin{array}{r}1,11 \\
10,99 \\
5,91 \\
5,66 \\
12,65 \\
3,00 \\
5,16 \\
7,24 \\
6,33 \\
2,83 \\
2,35 \\
5,05 \\
7,30 \\
6,05 \\
7,25 \\
2,35 \\
4,65\end{array}$ & $\begin{array}{c}0,85 \\
9,6 \\
5,44 \\
5,05 \\
11,12 \\
3,52 \\
4,80 \\
7,04 \\
6,00 \\
2,80 \\
2,16 \\
5,12 \\
6,72 \\
6,4 \\
6,08 \\
2,24 \\
4,16\end{array}$ & $\begin{array}{r}1,07 \\
11,15 \\
5,65 \\
5,20 \\
12,20 \\
3,60 \\
4,95 \\
7,45 \\
6,15 \\
2,85 \\
2,4 \\
4,85 \\
6,80 \\
6,40 \\
6,95 \\
2,10 \\
4,50\end{array}$ & $\begin{array}{c}0,44 \\
10,00 \\
5,65 \\
5,0 \\
11,40 \\
3,65 \\
5,15 \\
7,10 \\
6,10 \\
2,80 \\
2,15 \\
5,05 \\
7,10 \\
6,40 \\
6,20 \\
2,4 \\
4,65\end{array}$ \\
\hline $\begin{array}{l}\text { AAI } \ldots \ldots \ldots \ldots \ldots \ldots \\
\Sigma \text { AA } \ldots \ldots \ldots \ldots \ldots\end{array}$ & $\begin{array}{r}106,9 \\
55,58\end{array}$ & $\begin{array}{l}92,94 \\
51,81\end{array}$ & $\begin{array}{l}93,27 \\
53,72\end{array}$ & $\begin{array}{l}94,64 \\
52,34\end{array}$ \\
\hline Bilan azoté ${ }^{*} \ldots \ldots \ldots$ & 73,58 & 76,69 & 81,04 & 79,1 \\
\hline
\end{tabular}

TABLEAU 7

Ecart relatif moyen (ERM) de la composition en acides aminés des résidus entre les lots et avec les fèces et les bactéries ( $\left.N^{o s}\right)$.

Amino acids average relative difference ( $A R D)$ between insoluble faecal materials faeces and bacteries.

\begin{tabular}{|c|c|c|c|c|c|}
\hline & $N^{o s}$ & 8 & 9 & 10 & 11 \\
\hline $\begin{array}{l}\text { Résidu Frimas cru } \ldots \ldots \ldots \ldots \\
\text { Résidu Frimas cuit } \ldots \ldots \ldots \\
\text { Résidu Lincoln cru } \ldots \ldots \ldots \\
\text { Résidu Lincoln cuit } \ldots \ldots \ldots\end{array}$ & $\begin{array}{r}8 \\
9 \\
10 \\
11\end{array}$ & $\begin{array}{l}0 \\
8 \\
5 \\
6\end{array}$ & $\begin{array}{l}0 \\
6 \\
3\end{array}$ & $\begin{array}{l}0 \\
5\end{array}$ & 0 \\
\hline $\begin{array}{l}\text { Mélange bactéries } \ldots \ldots \ldots \ldots \\
\text { Fèces/Résidus correspondants . . . . }\end{array}$ & 5 & $\begin{array}{l}12 \\
21\end{array}$ & $\begin{array}{l}14 \\
26\end{array}$ & $\begin{array}{l}12 \\
24\end{array}$ & $\begin{array}{l}13 \\
33\end{array}$ \\
\hline
\end{tabular}


en proline des MAT insolubles des fèces du lot Lincoln est supérieure $(+20$ p. 100) à celle du lot Frimas. L'effet du traitement entraine aussi une diminution du total des teneurs en AA dans le cas des lots Frimas. Bien que les teneurs en proline, isoleucine, tyrosine aient augmenté de 6 à 12 p. 100 et que les teneurs en cystine et alanine restent inchangées, tous les autres acides aminés voient leur teneur diminuer de 5 à 12 p. 100 et jusqu'à -23 p. 100 pour le tryptophane. Dans le cas des lots Lincoln, le traitement n'a que peu d'effet sur les teneurs en thréonine, proline, valine, cystine, tyrosine et arginine. Par contre, l'effet de la cuisson se manifeste par une forte augmentation de la teneur en histidine (14 p. 100), ainsi que par une forte diminution des teneurs en tryptophane $(59 \mathrm{p} .100)$, en acide aspartique, lysine et méthionine (10 et $11 \mathrm{p} .100)$ et par une diminution plus faible des teneurs en acide glutamique, alanine et sérine (4 à 6 p. 100). Cependant, la cuisson des graines Lincoln n'entraîne pas de modification importante $(+1,5$ p. 100) du total des teneurs des 18 A.A. insolubles dosés dans les fèces.

La comparaison de la composition en acides aminés des résidus entre eux (tabl. 7) donne des valeurs d'ERM très faibles ( 3 à 8 ), ce qui indique une composition moyenne en acides aminés similaire pour les résidus des fèces des 4 lots étudiés. Les composés protéiques insolubles des fèces ont de plus une forte parenté avec le mélange référence des bactéries (ERM $=12$ à 14), ces valeurs étant nettement plus faibles que celles trouvées dans le cas de la comparaison bactéries/fèces totales (tabl. 5).

La comparaison entre fèces et résidus correspondants $(E R M=21$ à 33) montre que les matières azotées solubles ont une composition en acides aminés différente de celles des fèces.

\section{E. Composés azotés solubles des fèces}

\section{Importance des extraits solubles}

On a vu dans la figure 1 que les extraits solubles représentent une faible proportion de l'azote des fèces (19 et $16 \mathrm{p}$. 100), respectivement pour les lots Frimas et Lincoln. La fraction éthanolo soluble renferme toujours une proportion d'azote plus élevée que la fraction acide.

Cependant, l'effet traitement qui se manifeste par l'augmentation de scilibilité des composés azotés fécaux, est plus important dans la fraction acide, notamment pour le lot Lincoln.

\section{Matières azotées solubles (tabl. 8)}

Les extraits contiennent des composés (notamment des sucres aminés) qui interfèrent souvent avec le dosage de la phénylalanine et quelques fois le dosage de la tyrosine. Aussi, le bilan azoté des acides aminés des MA solubles dans l'éthanol est-il toujours très faible ( 37 à 45 p. 100) ce qui indique la présence dans cette fraction de composés azotés non dosés, en quantité importante. Dans la fraction acidosoluble le bilan azoté atteint 80 et 90 p. 100 , sauf dans le cas du lot Lincoln cru (54 p. 100). Le tryptophane n'a pas été dosé dans les extraits. 


\section{TABLEAU 8}

Composition en acides aminés ( $\mathrm{g} / 16 \mathrm{gN})$ des extraits solubles des fèces; comparaison entre les extraits (ERM).

Amino acid composition of faecal soluble extracts; $A R D$ between extracts.

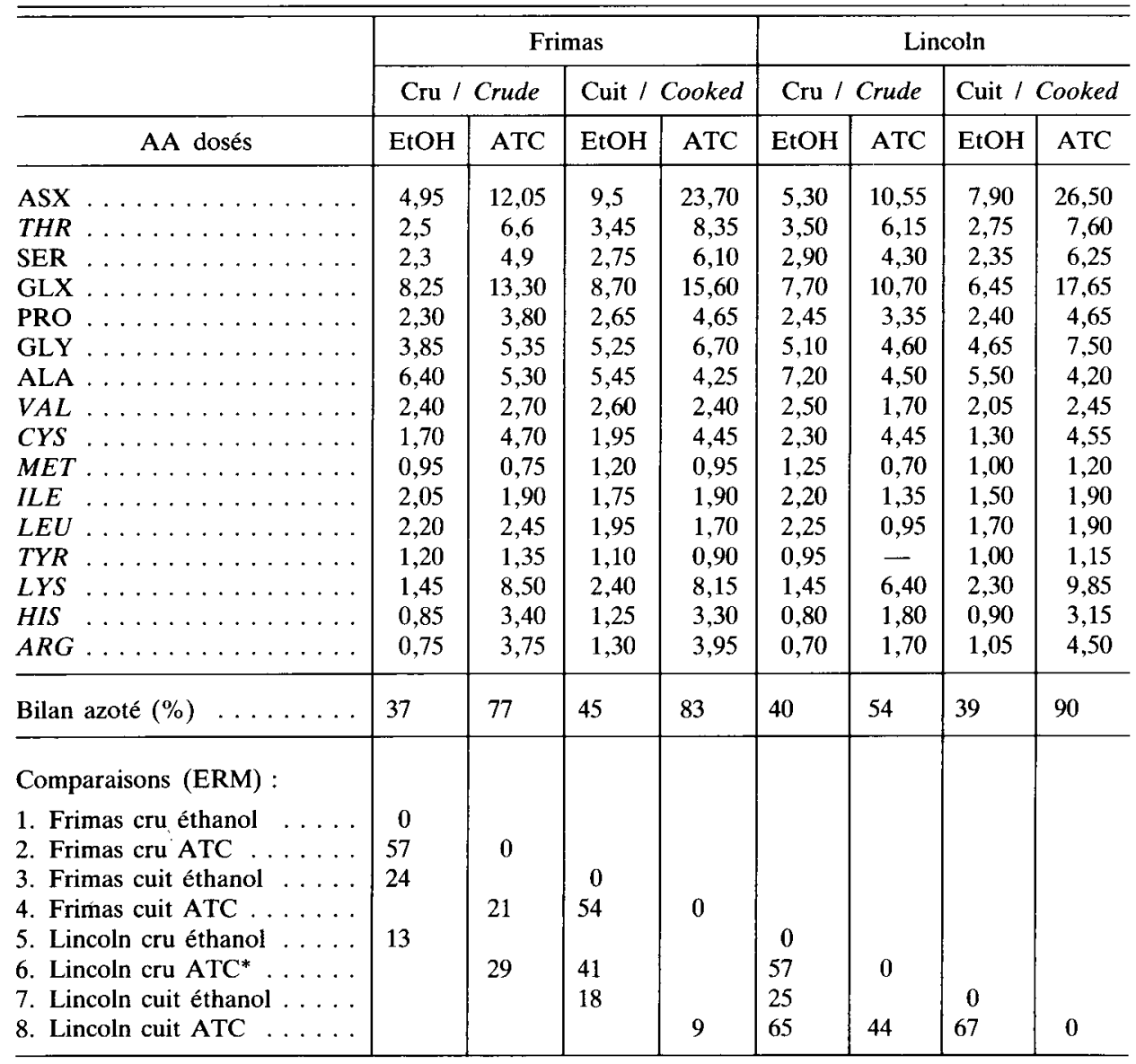

* Comparaison sans la tyrosine.

\section{Acides aminés solubles dans l'éthanol}

Les teneurs en acides aminés des extraits éthanoliques sont très faibles. Le total des teneurs des 16 acides aminés présentes dans le tableau 8 oscille entre 44 et $53 \mathrm{mg} /$ $100 \mathrm{~g}$ MAT, et les AAI comptent pour 36 p. 100 sauf dans le cas des Lincoln cuits où cette proportion n'atteint pas 35 p. 100 .

Quand on passe de l'utilisation de la variété Frimas à celle de la variété Lincoln, on note une augmentation importante des teneurs en thréonine (40 p. 100), en glycine 
(32 p. 100), en cystine (35 p. 100) ainsi qu'une augmentation plus légère des autres acides aminés sauf l'acide glutamique, l'histidine et l'arginine dont les teneurs accusent une faible diminution (6 p. 100).

L'effet du traitement se manifeste dans le cas de la variété Frimas par une diminution des teneurs en leucine et isoleucine ( -11 et 14 p. 100) alors que la teneur de tous les autres acides aminés est augmentée, quelquefois dans des proportions très importantes : 92 p. 100 pour l'acide aspartique, 73 p. 100 pour l'arginine, 65 p. 100 pour la lysine, 38 p. 100 pour la thréonine. Dans le cas de la variété Lincoln la cuisson entraîne une diminution $(-28$ p. 100) du total des AA dosés, laquelle atteint -43 p. 100 pour la cystine, -32 p. 100 pour l'isoleucine, -24 p. 100 pour la leucine et l'alanine. On observe en même temps une augmentation importante de la lysine $(+58$ p. 100) de l'acide aspartique $(+49$ p. 100) et de l'arginine (+ 35 p. 100).

\section{Acides aminés solubles dans l'ATC}

Les teneurs en acides aminés de cet extrait sont nettement plus élevées que dans le cas de l'extrait à l'éthanol avec 36 et 44 p. 100 d'AAI par rapport au total. Les teneurs en thréonine et sérine sont nettement plus élevées dans les extraits acides que dans la fraction éthanol et même dans la fraction insoluble.

Quand on passe de l'utilisation de la variété Frimas à la variété Lincoln, la teneur de tous les acides aminés de l'extrait ATC des fèces diminue quelquefois dans des proportions très importantes: histidine et arginine : $-47 \mathrm{p} .100$, isoleucine : -29 p. 100 , lysine : -25 p. 100 , acide aspartique : -12 p. 100 . Par contre, l'effet cuisson est moins homogène : dans le cas de la cuisson de la variété Frimas, on observe une augmentation très importante de la teneur en acide aspartique (97 p. 100) ainsi qu'une augmentation plus modeste des teneurs en thréonine (26 p. 100), proline (22 p. 100) glycine (25 p. 100), méthionine (26 p. 100) et arginine (21 p. 100). Ces variations sont accompagnées de diminution importante des teneurs en tyrosine leucine et sérine (33 à 24 p. 100). Dans le cas de la variété Lincoln, la cuisson a pour effet une faible diminution de la teneur en alanine $(6 \mathrm{p}$. 100) et une augmentation des teneurs de tous les autres acides aminés, quelquefois dans des proportions considérables : pour l'acide aspartique : 150 p. 100 , pour l'arginine : 165 p. 100 , pour l'histidine : 75 p. 100 , la méthionine : 71 p. 100 , l'acide glutamique : 65 p. 100 .

\section{Comparaison de la composition en acides aminés des extraits solubles}

Ces caractéristiques se retrouvent lorsque l'on compare (bas du tableau 8) la composition en acides aminés des fractions solubles entre elles. Il y a en effet une parenté assez homogène entre les fractions éthanosolubles (ERM = 13 à 25). Dans le cas des composés acido solubles les valeurs de ERM sont plus échelonnées : l'écart le plus faible est observé entre les lots cuits Frimas et Lincoln (ERM =9). L'écart le plus élevé $(E R M=44)$ concerne l'effet du traitement dans le cas des lots Lincoln. Le calcul de l'ERM entre les fractions solubles dans l'éthanol et dans l'ATC donne des valeurs nettement plus élevées (41 à 69). Ceci confirme que ces composés sont de nature différente. 


\section{F. Estimation de la taille des composés aminés solubles (fig. 3)}

Dans le cas des lots Lincoln, où une augmentation importante des proportions d'azote soluble fécal a été constatée dans le cas de l'ingestion des graines cuites, le passage sur séphadex G 25 des extraits éthanol et acido solubles a permis de séparer plusieurs classes de composés.

Dans l'éthanol on ne retrouve qu'un seul pic situé dans la zone des faibles poids moléculaires. Les composés présents dans ce pic contiennent en moyenne moins de 2 résidus d'acides aminés. Ce pic étalé sur 15 fractions, dans le cas de l'ingéré cru correspond à des peptides dont la concentration est évaluée à $220 \mathrm{mg} \mathrm{N}$ pour $100 \mathrm{~g}$ de fèces sèches. L'effet de la cuisson se traduit par un resserrement du pic (6 fractions) et une augmentation de 100 p. 100 de sa teneur en azote aminé, mais le nombre de résidus d'acides aminés ne semble pas modifié.
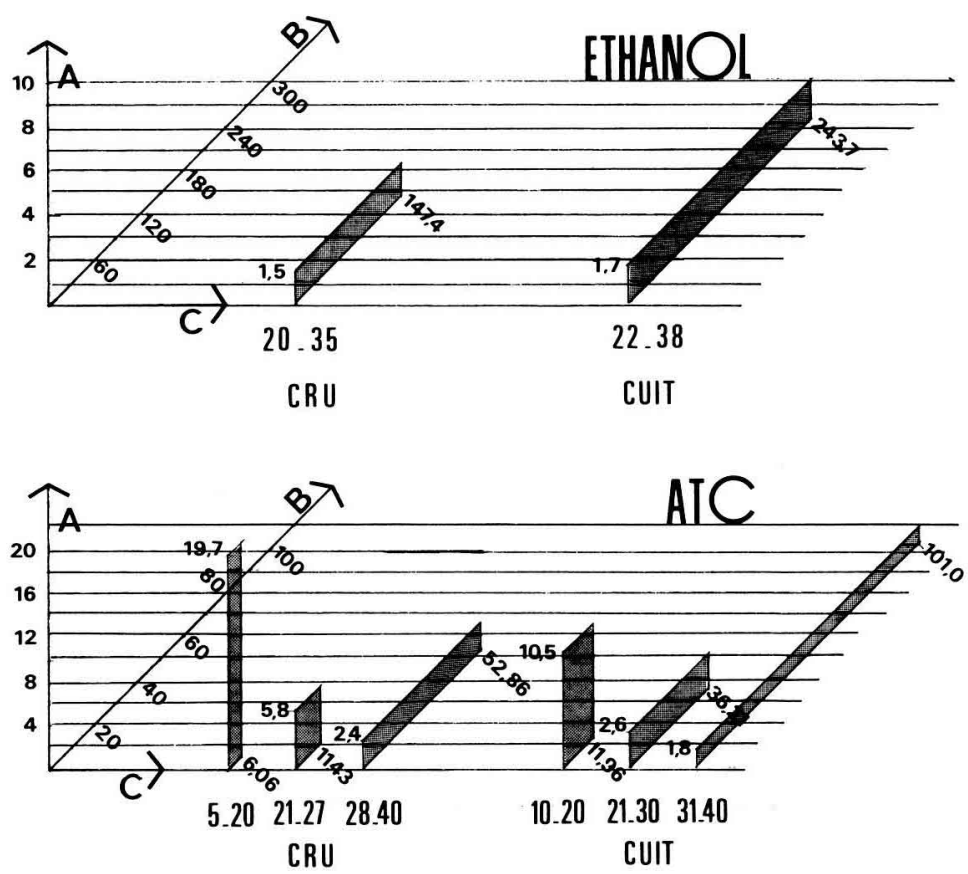

FIG. 3

Influence de la cuisson des graines Lincoln ingérées par les rats sur les peptides solubles de leurs fèces.

Amino Nitrogen and Size of Faecal soluble Fractions from rats fed Lincoln raw/cooked peas.

\footnotetext{
A : Estimation du nombre de résidus d'AA dans chaque fraction $=\mathbf{B}^{\prime} / \mathbf{B}$. estimated number of $A A$ residues in each fraction $\left(=B^{\prime} / B\right)$.

$\mathrm{B}:$ [N aminé libre] $\mathrm{g} / 100 \mathrm{~g}$ fèces.

[free amino $N] \mathrm{g} / 100 \mathrm{~g}$ faeces.

$\mathrm{B}^{\prime}$ : [N aminé total] $\mathrm{g} / 100 \mathrm{~g}$ fèces; représenté par l'aire grisée $(=\mathrm{B} \times \mathrm{A})$. [total amino $N$ ] $\mathrm{g} / 100 \mathrm{~g}$ faeces, signalled by the grey area $(=B \cdot A)$.

$\mathrm{C}$ : fractions recueillies pour chaque pic élué après chromato $\mathrm{G} 25$. eluted peaks from Sephadex $G 25$.
} 
Dans les extraits ATC, on a séparé 3 familles de composés. La première famille est éluée dans la zone des poids moléculaires élevés. Dans le cas du lot Lincoln cru il contiendrait 20 résidus d'acides aminés et une concentration en azote aminé de $119 \mathrm{mg} /$ $100 \mathrm{~g}$ de fèces sèches. La cuisson ne modifie pas la concentration en azote aminé de ce pic, mais diminue de moitié l'estimation du nombre de résidus d'AA retrouvés. La deuxième famille contient des composés de poids moléculaire plus faible et environ 6 résidus d'acides aminés avec une concentration en azote aminé de $67 \mathrm{mg} / 100 \mathrm{~g}$ de fèces sèches. L'effet de la cuisson se traduit par une augmentation de $40 \mathrm{p}$. 100 de la teneur en azote aminé et par une diminution de moitié du nombre des résidus d'acides aminés. La troisième famille est éluée dans la zone des plus faibles poids moléculaires ; il ne contient que 2 résidus d'acides aminés en moyenne et sa teneur en azote aminé est un peu plus élevée que celle de la première famille. La cuisson se manifeste par une augmentation de 30 p. 100 de la concentration en azote aminé, mais ne modifie pas de façon sensible le nombre des résidus d'acides aminés.

\section{Discussion}

Les rats ayant ingéré les régimes à base de pois présentent une augmentation du poids du coecum plein par rapport aux cocums de rats ayant ingéré des régimes à base de farine de viande, ou de caséine. Ceci correspond à une accumulation de matériel non digéré à la fin de l'intestin grêle. Dans le cas des lots Lincoln, cette accumulation est un peu plus élevée que dans le cas des lots Frimas, et correspond à une ingestion plus importante de cellulose, de raffinose, de stachyose dont l'effet est amplifié par la cuisson. En effet, à partir de la composition globale des régimes et de la composition glucidique des graines de pois lisses et ridées (Cerning-Béroard \& Filiatre-Verel, 1979) on peut estimer que $100 \mathrm{~g}$ MS ingérées apportent dans le cas des lots Frimas et Lincoln respectivement : 3,3 et $4,7 \mathrm{~g}$ de cellulose, 1,4 et $1,9 \mathrm{~g}$ de saccharose, 0,4 et $1,33 \mathrm{~g}$ de raffinose, 2,41 et $2,35 \mathrm{~g}$ de verbascose, 1,7 et $2,35 \mathrm{~g}$ de stachyose ainsi que 29,5 et $18 \mathrm{~g}$ d'amidon provenant des graines. Or, l'amidon des graines de pois ridés contient une proportion nettement plus élevée d'amylose que celui des graines de pois lisses (70,9 et 33,2 p. 100) selon Colonna \& Mercier (1984). De plus, l'amylose des graines ridées est de type $\mathrm{B}$, dont la réorganisation des chaînes en milieu aqueux donne des composés très indigestibles (CHAMP, 1985). L'accumulation de ces glucides réputés indigestibles peut expliquer l'augmentation du poids des contenus du cœcum et du gros intestin des rats.

Les CUDA de la MAT des pois sont un peu plus élevées que celles citées par Pron et al. (1979). Toutefois, ces auteurs avaient noté que la cuisson des graines entraînait une baisse du CUDA de la MAT du pois lisse. Les valeurs du CUDA de la MAT des légumineuses semblent influencées par les autres constituants des graines. Pour la féverole, le CUDA de la MAT est de 70 p. 100 dans le cas de la variété S.45 riche en tanins et de 80 p. 100 pour la variété Bianka sans tanin ; pour le Lupin jaune, le CUDA de la MAT est de 84 p. 100 (PION et al., 1979) et tombe à 77,6 p. 100 pour la variété multolupa (Prieto \& Aguilera, 1986). Pour la lentille, le CUDA de la MAT est de 76 p. 100 (Sarwar \& Peace, 1986).

La fraction insoluble des fèces contient surtout des acides aminés dont la composition est très voisine de celle du mélange des bactéries dont le développement est permis 
par la présence de glucides alimentaires digestibles non dégradés dans l'iléon accompagnés des sécrétions digestives endogènes. Le changement de variété et le traitement ont peu d'effet sur la composition en acides aminés du résidu insoluble des fèces. Cependant, la teneur en proline est plus élevée dans le cas de l'utilisation du lot Lincoln que dans celui du lot Frimas. La cuisson entraîne une diminution importante des teneurs en tryptophane qui est plus sensible dans le cas du lot Lincoln.

Les fractions solubles contiennent une faible part de l'azote fécal mais celle-ci est fortement augmentée par le traitement des graines, surtout dans le cas des lots Lincoln. Les proportions d'azote soluble n'atteignent cependant jamais celles retrouvées après ingestion de régime à base de caséine, dans les contenus de coecum des rats holoxéniques (43 à 53 p. 100) ou axéniques ( 73 à 89 p. 100). Dans ce dernier cas la contribution des bactéries aux phénomènes de digestion était évidemment nulle (COMBE et al, 1970).

Le CUDA des AA est toujours un peu plus élevé que celui des MAT et varie dans une plus faible mesure. Dans le cas de l'utilisation de la fèverole Ascot décortiquée et micronisée, l'alanine, la valine, la tyrosine et la cystine ont des CUDA nettement inférieurs alors que l'arginine la phénylalanine et l'histidine ont des CUDA supérieurs à la moyenne des AA et au CUDA des MAT (MENDÈs-Pereira et al., 1977). Sur la base de la composition en acides aminés, ces auteurs avaient aussi montré que les fèces différaient davantage de l'aliment que des bactéries ou de l'endogène, sans toutefois pouvoir distinguer la part des bactéries de celles de l'endogène. Darcy et al. (1982) ont montré chez le porc que la digestibilité fécale apparente de l'azote était un mauvais critère de prédiction de la digestibilité des AA des régimes de poisson. Ils considèrent que la dispersion des CUDA des différents AAI est si importante qu'elle ne permet pas d'envisager d'utiliser le CUDA moyen des AA en remplacement. Ceci correspond bien à nos résultats, notamment pour les acides aminés soufrés et aussi pour le tryptophane. Sève et al. (1985) estiment à 83,7 et 84,1 p. 100 la digestibilité des protéines de pois (Var. Finale) crus et traités chez le porcelet recevant 30 p. 100 de sa ration protéique sous cette forme. Ceci est en accord avec nos résultats, notamment pour les graines crues.

Nos résultats concernant les sucres aminés présents dans les fèces sont à rapprocher de ceux publiés antérieurement (COMBE et al, 1980). Dans les fèces de rats ayant ingéré un régime à base de caséine, les teneurs en glucosamine et galactosamine sont deux fois plus faibles mais le rapport des concentrations est également de 2,6. Dans tous les autres cas (fèces provenant de rats ou d'agneaux axéniques et contenus digestifs de rats ou de veaux) le rapport des concentrations glucosamine/galactosamine caractérisé comme représentatif de l'endogène est nettement inférieur à 1,9 et correspond davantage au cas observé pour les fèces des rats ayant ingéré les pois Lincoln cuits. Les fèces provenant du lot Lincoln cuit sont ceux qui contiendraient la part d'endogène la plus élevée.

Les composés azotés solubles sont de faible taille : acides aminés libres et dipeptides seulement dans la fraction éthanol. On trouve des composés de taille un peu plus élevée dans la fraction acidosoluble. Ceci avait déjà été mentionné par Pıon et al. (1971). L'extrait éthanolique contient les composés azotés du catabolisme des acides aminés et des protéines, et proportionnellement peu d'acides aminés, bien que l'effet du traitement se manifeste par une augmentation des teneurs en lysine, aginine et acide aspartique dans cette fraction. La fraction acido soluble contient toujours les teneurs les plus élevées en sérine et thréonine et sa composition en acides aminés est moins influencée par les paramètres étudiés que la fraction éthanolosoluble ; la proportion de substances azotées d'origine endogène pourrait donc être notable. 


\section{Conclusion}

Bien que les valeurs du CUDA des MAT des graines de Pois Lincoln et Frimas soient semblables, les valeurs du CUDA des AA des graines Frimas sont nettement plus faibles que celles des graines Lincoln. De plus le CUDA des acides aminés soufrés et du tryptophane est toujours nettement inférieur à celui de l'ensemble des AA et même à celui de l'azote. Ceci montre l'intérêt de la détermination de ces coefficients dans le cas où ces acides aminés indispensables sont limitants dans la ration. L'analyse de la composition azotée des fèces des rats ayant ingéré ces graines permet de penser que les processus de digestion sont fortement influencés par la composition glucidique de la ration.

La fraction insoluble des fèces semble correspondre aux bactéries dont le développement se trouve favorisé par l'ingestion d'une ration plus riche en cellulose, raffinose et amylose (cas des lots Lincoln), ou par le traitement à l'autoclave des graines, qui modifie la structure des amidons. Ainsi, les glucides présents dans la partie distale du tube digestif peuvent être utilisés par les bactéries. Par contre, la sensibilité de la teneur en tryptophane des MAT insolubles fécales aux paramètres étudiés met l'accent sur une composante d'origine alimentaire.

Les fractions solubles contiennent des composés en voie d'hydrolyse qui présentent encore les caractéristiques de certains composés d'origine endogène (sucres aminés, sérine, thréonine peu modifiés par les paramètres étudiés) ou qui, très sensibles aux paramètres étudiés (composition glucidique de la ration, effet du traitement) auraient plutôt une origine alimentaire, directe ou indirecte.

Ces résultats illustrent les remaniements de la microflore dans le cœcum et le gros intestin à partir des substrats d'origine alimentaire ou endogène.

Reçu en janvier 1988.

Accepté en décembre 1988.

\section{Remerciements}

Nous remercions J. Prugnaud qui a assumé la responsabilité du bon fonctionnement des appareils de dosage automatique des acides aminés et G. BAYLE qui a réalisé le dosage des sucres aminés.

\section{Summary}

Effects of variety and steam cooking of peas (Pisum sativum L.) on nitrogen digestion in the rat

The aim of this study was to investigate nitrogen digestion of pea seeds in growing rats. The effect of amino-acid and carbohydrate concentration of the seeds was studied using Lincoln and 
Frimas varieties of steam-cooked or raw seeds as sole source of protein in 4 balanced experimental diets ( 15 p. 100 total crude protein and 11000 Kilo-Joules $/ \mathrm{kg}$ dry matter).

In raw pea diets nitrogen apparent digestibility (NAD) was 85 p. 100 for both varieties, but that of some AA was much lower. When the seeds were steam cooked the NAD of the Frimas variety fell to $82.6 \mathrm{p} .100$ and that of the Lincoln variety to $76.6 \mathrm{p} .100$.

The nature and concentration of nitrogen compounds in the faeces of the 4 experimental groups were different, suggesting that the digestive processes were different. Glucosamine concentrations in faecal proteins were $3.5 \mathrm{~g} / 16 \mathrm{gN}$ in all cases except that of cooked Frimas seeds when the concentration increased by 31 p. 100 . Galactosamine concentrations were half that of glucosamine in the faecal proteins of rats fed the raw seeds. Steam cooking increased galactosamine concentrations by 43 and 55 p. 100 in Frimas and Lincoln seeds respectively.

Soluble fractions contained $16-19 \mathrm{p} .100$ of total faecal nitrogen with the raw diet ; steam cooking increased this proportion to 26 and $31 \mathrm{p} .100$. Ethanol soluble nitrogen compounds showed low molecular weights, and low amino acid concentrations, which were very sensitive to the effects of variety and steam cooking. Trichloracetic soluble nitrogen compounds appeared mostly as peptides, proteins and mucoproteins. These soluble compounds, which are partially hydrolysed, show dietary and endogenous characteristics.

The faecal residue fractions from the 4 experimental assays had a very similar amino acid composition to that of bacteria. However, the dry weight of faecal residue was higher when Lincoln variety and steam cooking were used.

In conclusion, the effects of steam cooking of pea seeds and of dietary carbohydrate contents (cellulose, raffinose and amylose) enhanced the development of the intestinal flora and the digestive activity.

Key words : Faeces, rat, pea seeds, effects of variety, effect of treatment.

\section{Références bibliographiques}

Cerning-Beroard J., Filiatre-Verel A., 1979. Etude comparée de la composition glucidique des graines de Pois Lisse et Ridé. Lebensm.-Wiss.-Technol., 12, 273-280.

Champ M., 1985. Digestion des glucides chez le monogastrique. Rep. Nutr. Dévelop., 25, 819-842.

Colonna P., Mercier C., 1979. Les amidons de Légumineuses. Aspect, composition, structure et propriétés physico-chimiques. Lebensm.-Wiss.-Technol., 12, 1-12.

Combe E., Pion R., SAcquet E., 1970. Influence de la nature et du taux des protéines alimentaires sur la composition en acides aminés du contenu du cœcum du rat axénique. Ann. Biol. anim. Bioch. Biophys., 10, 697-702.

Combe E., Patureau-Mirand P., Bayle G., Pion R., 1980. Influence de l'aliment et de la microflore sur la teneur en sucres aminés des contenus digestifs et des fèces chez le rat, l'agneau et le veau préruminant. Reprod. Nutr. Develop., 20, 1707-1715.

Darcy B., Laplace J.P., Duee P.M., 1982. Digestion des protéines dans l'intestin grêle chez le porc. 1. - Digestibilité des acides aminés selon la source des protéines d'un régime à base d'amidon de maïs purifié. Ann. Zootech., 31, 275-300.

Eggum B.O., Beames R.M., Bach-KNusSEn K.E., 1986. The effect of varying crude protein level and the proportions of fibre in diets containing a mixture of meat and bone meal and wheat bran as the only protein source on nitrogen balances indices and energy digestibility in the rat. Br. J. Nutr., 56, 429-458.

FLeming S.E., 1981. Influence of cooking methods on digestibility of legume and cereal starches. J. Food Sci., 47, 1-3.

Mason V.C., Just A., Bech-ANDERSEN S., 1976. Bacterial activity in the hind-gut of pigs 2. Its influence on the apparent digestibility of nitrogen and amino acids. Z. Tierphysiol., Tierernähr. Futtermittelkde, 36, 310-324. 
Mendes-Pereira E., Pion R., Prugnaud J., 1977. Utilisation digestive apparente des acides aminés de quelques aliments chez le rat en croissance. Ann. Biol. anim. Bioch. Biophys., 17, 625-631.

Mosse J., Huet J.C., Baudet J., 1987. Changements de la composition en acides aminés des graines de pois en fonction de leur taux d'azote. Sci. Aliments, 7, 301-324.

Moores S., Spackmann D.M., Stein W.M., 1958. Chromatography of amino acids on sulfonated polystyrene resins. Anal. Chem., 30, 1185-1190.

Pion R., Valenza C., Combe E., 1971. Les matières azotées des contenus de cœcum de rat : méthode d'étude et résultats obtenus : influence de l'état axénique. Ann. Biol. anim. Bioch. Biophys., 11, 337-338.

Pion R., Patureau-Mirand P., Combe E., Mendes-Pereira E., 1977. The digestibility of amino acids of different feeds for rats, lambs and non ruminating calves. II ${ }^{\text {md }}$ International symposium on protein metabolism and nutrition. Flevohof 1977, E.A.A.P. 76-78.

Pion R., Mendes-Pereira E., Prugnaud J., 1979. Effect of composition and process on the nutritive value of some legume seeds. J. Am. Oil Chemist. Soc., 56, 150-153.

Prieto C., Agullera J.F., 1986. The effect of the supplementation with methionine and lysine of diets based on lupin seed (Lupinus albus var. multolupa.). J. Anim. Physiol. Anim. Nutr., 55, 239-246.

Reichert D.R., MacKenzie S.L., 1982. Composition of peas (Pisum sativum) varying widely in protein contents. Agric. Food Chemist., 30, 312-317.

Sarwar G., Peace R.W., 1986. Composition between true digestibility of total nutrogen and limiting amino acids in vegetable proteins fed to rats. J. Nutr., 116, 1172-1184.

Seve B., Aumaitre A., Bouchez P., 1985. Effet d'un traitement technologique sur la valeur nutritionnelle du pois pour le jeune porcelet. Sci. Aliments, 5, 119-126.

Slump P., Schreuder M.A.W., 1969. Determination of tryptophan in foods. Anal. Biochem., 27, 182-186. 